\title{
Splenic Laceration After Endoscopic Retrograde Cholangiopancreatography
}

\author{
Emad Geddoa, MRCS, Nafees A. Qureshi, MRCS, Darren K. Patten, MRCS, Meher Lad, MBBS, \\ Sarah Duncan, MBBS, Neil Foden, MRCP, David L. Stoker, FRCS \\ Department of General Surgery, North Middlesex University Hospital, London, England (Mr. Geddoa, Dr. Qureshi, Mr. \\ Patten, Dr. Lad, Dr. Duncan, Mr. Stoker). \\ Department of Biosurgery and Surgical Oncology, St Mary's Hospital, Imperial College Healthcare NHS Trust, London, \\ England (Mr. Patten).
}

\begin{abstract}
The advent of endoscopic retrograde cholangio-pancreatography (ERCP) has enabled physicians and surgeons to carry out a range of diagnostic and therapeutic procedures pertaining to various pathologies found within the hepato-biliary system. Despite being relatively less invasive when compared to traditional methods of surgery, ERCP still carries recognized complications that are a cause for morbidity amongst patients. Furthermore, rarer complications can occur in difficult circumstances that are potentially fatal. We report two cases whereby the patients sustained serious splenic injuries as a consequence of the procedure. Splenic lacerations were diagnosed on computer tomography scanning in both patients who subsequently underwent emergency splenectomy. The patients made an uneventful recovery and returned home after surgery.

To date twelve cases have been reported in current medical literature regarding injury to the spleen following ERCP. These aforementioned injuries have arisen particularly when the endoscopist has encountered difficult or variations in anatomy whilst performing ERCP. In addition to presenting two cases, we review the literature and discuss the implications of these complications and how this may affect the way in which clinicians take informed consent from patients before conducting ERCP.

Citation Geddoa E, Qureshi NA, Patten DK, Lad M, Duncan S, Foden N, Stoker DL. Splenic laceration after endoscopic retrograde cholangiopancreatography. CRSLS e2014.001763. DOI: 10.4293/CRSLS.2014.001763.

Copyright (C) 2014 SLS This is an open-access article distributed under the terms of the Creative Commons Attribution-Noncommercial-ShareAlike 3.0 Unported license, which permits unrestricted noncommercial use, distribution, and reproduction in any medium, provided the original author and source are credited.

Address correspondence to: Darren K. Patten, MRCS, Department of Biosurgery and Surgical Oncology, St Mary's Hospital, Imperial College Healthcare NHS Trust, London, W2 1NY, England. Telephone: 0208887 2000, Fax: 0208887 4219, E-mail: Darren.patten@gmail.com
\end{abstract}

\section{INTRODUCTION}

Endoscopic retrograde cholangiopancreatography (ERCP) plays an important role in the management of hepatobiliary and pancreatic disorders. Since its introduction in 1970, ERCP has been reported to be associated with certain common complications and some uncommon complications, including acute pancreatitis, common bile and pancreatic ductal injury, and gastric/duodenal perforation. ${ }^{1}$ Splenic trauma is an extremely rare and potentially fatal complication of ERCP, and only 12 cases have been reported in the English-language medical literature since 1988 (Table 1). However, this uncommon but serious complication deserves the attention of general surgeons and gastroenterologists who are involved in performing this procedure.
In this case series, we present 2 cases of post-ERCP splenic trauma occurring as a result of ERCP; the patients represented a few hours after ERCP with an acute abdomen and subsequently underwent laparotomy with splenectomy for splenic injury.

\section{CASE REPORT}

\section{Case 1}

The first patient was a 75-year-old man with a long history of epigastric pain. His comorbidities included hypertension, hypercholesterolemia, and an abdominal aortic aneurysm measuring $4.75 \mathrm{~cm}$ in diameter. He had undergone multiple esophagogastroduodenoscopies (OGDs) between 1998 and 2005 with no significant findings but 
Table 1.

Reported Cases of Splenic Injury After ERCP

\begin{tabular}{|c|c|c|c|}
\hline Author & Patient & Indication & Outcome \\
\hline Lo et $\mathrm{al}^{3}$ & $79 \mathrm{y}$, man & Ascending cholangitis & Splenic subcapsular hematoma \\
\hline Wu et $\mathrm{al}^{5}$ & $57 \mathrm{y}$, woman & Abdominal pain and abnormal LFTs & Splenic avulsion from capsule and liver hematoma \\
\hline Lewis et $\mathrm{al}^{6}$ & $63 \mathrm{y}$, woman & Ascending cholangitis and pancreatic mass & Avulsion of short gastric vessels \\
\hline Trondsen et $\mathrm{al}^{8}$ & $46 \mathrm{y}$, woman & Acute pancreatitis & Splenic rupture \\
\hline Cho et $\mathrm{al}^{9}$ & $63 \mathrm{y}$, woman & Cholecystitis & Splenic laceration \\
\hline Gaffney et $\mathrm{al}^{10}$ & $48 \mathrm{y}, \operatorname{man}$ & CBD stent exchange & Splenic laceration and subcapsular hematoma \\
\hline Ong et $\mathrm{al}^{11}$ & $55 \mathrm{y}$, woman & Intermittent epigastric pain & Splenic laceration \\
\hline Badaoui et $\mathrm{al}^{12}$ & $42 \mathrm{y}, \operatorname{man}$ & Epigastric pain and dilated CBD & Splenic laceration and hemoperitoneum \\
\hline
\end{tabular}

LFTs $=$ liver function tests.

was found to have inflammatory pyloric stenosis on an OGD in October 2010 (confirmed on histologic analysis). During examination, he was found to have Calculi in the gallbladder and common bile duct (CBD). He underwent OGD for dilatation of pyloric stenosis followed by an ERCP with sphincterotomy and stenting of the CBD in April 2011. ERCP proved to be a challenging procedure because of looping of the endoscope in the stomach and a subsequent inability to reduce the loop because the stomach was "too baggy." A cholangiogram confirmed a Calculus in the CBD, but it could not be removed because the patient became restless during the procedure. He was kept overnight in the hospital because he was complaining of severe abdominal pain. An urgent computed tomography (CT) scan of the abdomen after a surgical consultation excluded bowel perforation or any other cause of abdominal pain. A repeat OGD for pyloric dilatation and ERCP were performed 1 month later. For sedation, midazolam, $5 \mathrm{mg}$; diazepam, $10 \mathrm{mg}$; butylscopolamine, $20 \mathrm{mg}$; and fentanyl, $100 \mathrm{mg}$, were used. The endoscopes used were TJF 240 S/N 2030285 and TGF 240-2900223 Colymp. The pyloric stenosis was dilated with a balloon to $18 \mathrm{~mm}$. With some difficulty, the TJF endoscope was advanced to the second part of the duodenum (D2) with a large gastric loop. Balloon trawls yielded a solitary CBD Calculus. The duct was clear at the end of the procedure, thus requiring no further stenting. The patient was kept in the hospital under observation for 4 hours after the procedure and was discharged home.
The patient felt unwell during the night with symptoms of dizziness and called for an ambulance in the early hours of the morning. He was found to have an altered level of consciousness. He was in a state of shock on arrival to the hospital (hypotensive and tachycardic). After fluid resuscitation in the accident and emergency (A\&E) department, an urgent CT angiogram was arranged to rule out a suspected abdominal aortic aneurysm rupture.

Blood tests showed a hemoglobin level of $10.0 \mathrm{~g} / \mathrm{dL}$ and platelet count of $239 \times 10^{9} / \mathrm{L}$. Inflammatory markers were raised (white cell count, $6.1 \times 10^{9} / \mathrm{L}$; neutrophil count, 4.4 [72.1\%] $\times 10^{9} / \mathrm{L}$; C-reactive protein level, $3 \mathrm{mg} / \mathrm{L}$ ); renal function tests showed abnormalities (urea level, 2.6 $\mathrm{mmol} / \mathrm{L}$; creatinine level, $131 \mu \mathrm{mol} / \mathrm{L})$. In addition, liver function tests showed normal findings.

A CT scan showed splenic rupture and free fluid in the abdomen (Figure 1). The patient underwent an emergency splenectomy within 2 hours. The intraoperative findings were a capsular tear with a large amount of intraperitoneal blood but no injury to the splenic hilum. There was no associated bowel injury. Histology reported that the spleen weighed $47 \mathrm{~g}$ and measured $75 \times$ $60 \times 25 \mathrm{~mm}$. A small defect in the capsule, in keeping with a splenic capsular rupture, measured $13 \mathrm{~mm}$. The white pulp of the spleen showed normal vessel architecture, and moderate lymphovascular hyperplasia was noted. The red pulp was found to be congested with no evidence of hematopoiesis. Furthermore, there was no evidence of malignancy within the specimen. Postop- 


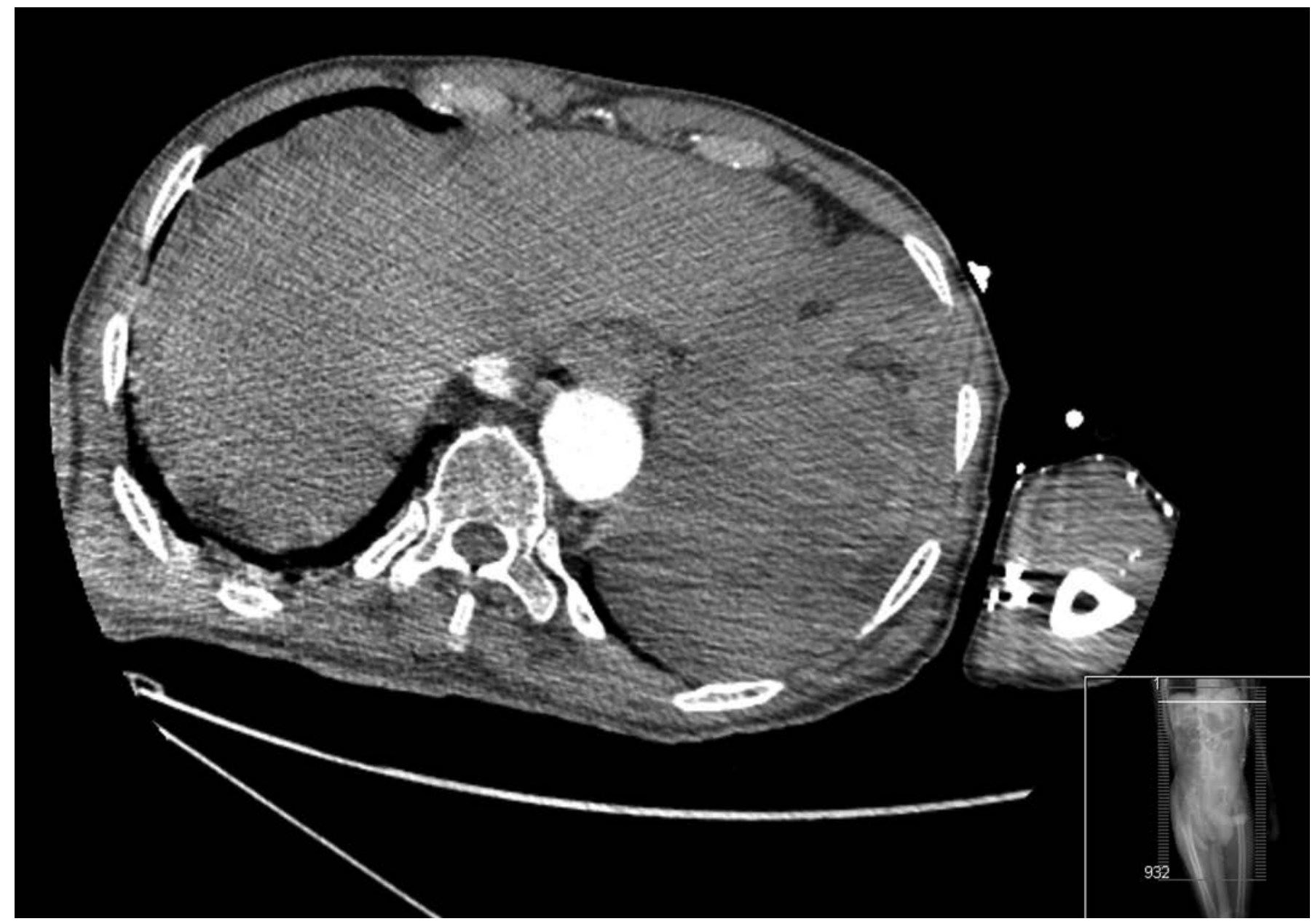

Figure 1. CT angiogram of patient 1 showing splenic enlargement with heterogeneous density suggesting splenic rupture.

eratively, the patient was admitted to the intensive therapy unit for 48 hours and made an uneventful recovery. He received post-splenectomy vaccines and was discharged home 7 days postoperatively receiving lifelong penicillin $\mathrm{V}$, with a follow-up appointment in the outpatient clinic.

At the 3-month clinic follow-up, the patient did not complain of gallstone-related abdominal pain and an ultrasonography scan was performed; it showed one 9-mm gallstone within the gallbladder, but gallstones could be seen within the CBD. In light of his asymptomatic gallstone disease, the patient refused to undergo a laparoscopic cholecystectomy.

\section{Case 2}

A 72-year-old woman underwent ERCP for CBD Calculi. The ERCP was recorded as difficult because the patient was restless during the procedure, but no technical difficulties were encountered and the procedure was completed without any acute on-table complications. She initially had an acute onset of abdominal pain starting soon after her ERCP procedure. The endoscopy team arranged an urgent CT scan of the abdomen with intravenous contrast; it only showed rectal wall thickening, which was the only positive finding. No acute cause of her abdominal pain, such as bleeding, blushing, or perforation, was found. She was discharged home the same day with analgesia. However, the patient's abdominal pain worsened over the course of 24 hours after ERCP, causing her to present to the A\&E department. The pain was dull and constant and was associated with nausea and vomiting. On presentation to the A\&E department, the patient was hemodynamically stable. Abdominal examination showed mild generalized tenderness, more so in the left upper quadrant, with muscular 
guarding and evidence of peritonism. Findings of a digital rectal examination were normal. The patient had a medical history of alcohol abuse, smoking, and left-sided pneumonia (3 months before ERCP).

Blood tests showed a hemoglobin level of $11.4 \mathrm{~g} / \mathrm{dL}$ and platelet count of $404 \times 10^{9} / \mathrm{L}$. Inflammatory markers were raised (white cell count, $20.4 \times 10^{9} / \mathrm{L}$; neutrophil count, $17.3[84.8 \%] \times 10^{9} / \mathrm{L}$; C-reactive protein level, $28 \mathrm{mg} / \mathrm{L}$; renal function tests showed slight abnormalities (urea level, $9.3 \mathrm{mmol} / \mathrm{L}$; creatinine level, $112 \mu \mathrm{mol} / \mathrm{L}$ ). Findings of liver function tests were normal apart from a raised bilirubin level ( $29 \mu \mathrm{mol} / \mathrm{L})$. An urgent CT scan with intravenous contrast of the abdomen was arranged, 1 hour after the patient's presentation to the A\&E department, which showed free intraperitoneal fluid and a ruptured spleen (Figure 2). After discussion with the on-call radiologist and consultant surgeon, it was decided to proceed with an emergency laparotomy and splenectomy because there was no local interventional radiology facility to perform splenic artery embolization. During laparotomy, large amounts of free blood and blood clots were noted. The spleen was found to have a full-thickness laceration at the inferior pole with active blood oozing. There was no vascular injury at the hilum of the spleen. Despite thorough review of the images from the first CT scan, which was performed 24 hours before the patient presented to the A\&E department, there was no CT indication suggesting splenic injury.

The specimen sent for histologic analysis weighed $47 \mathrm{~g}$ and measured $100 \times 54 \times 22 \mathrm{~mm}$. The capsule of the

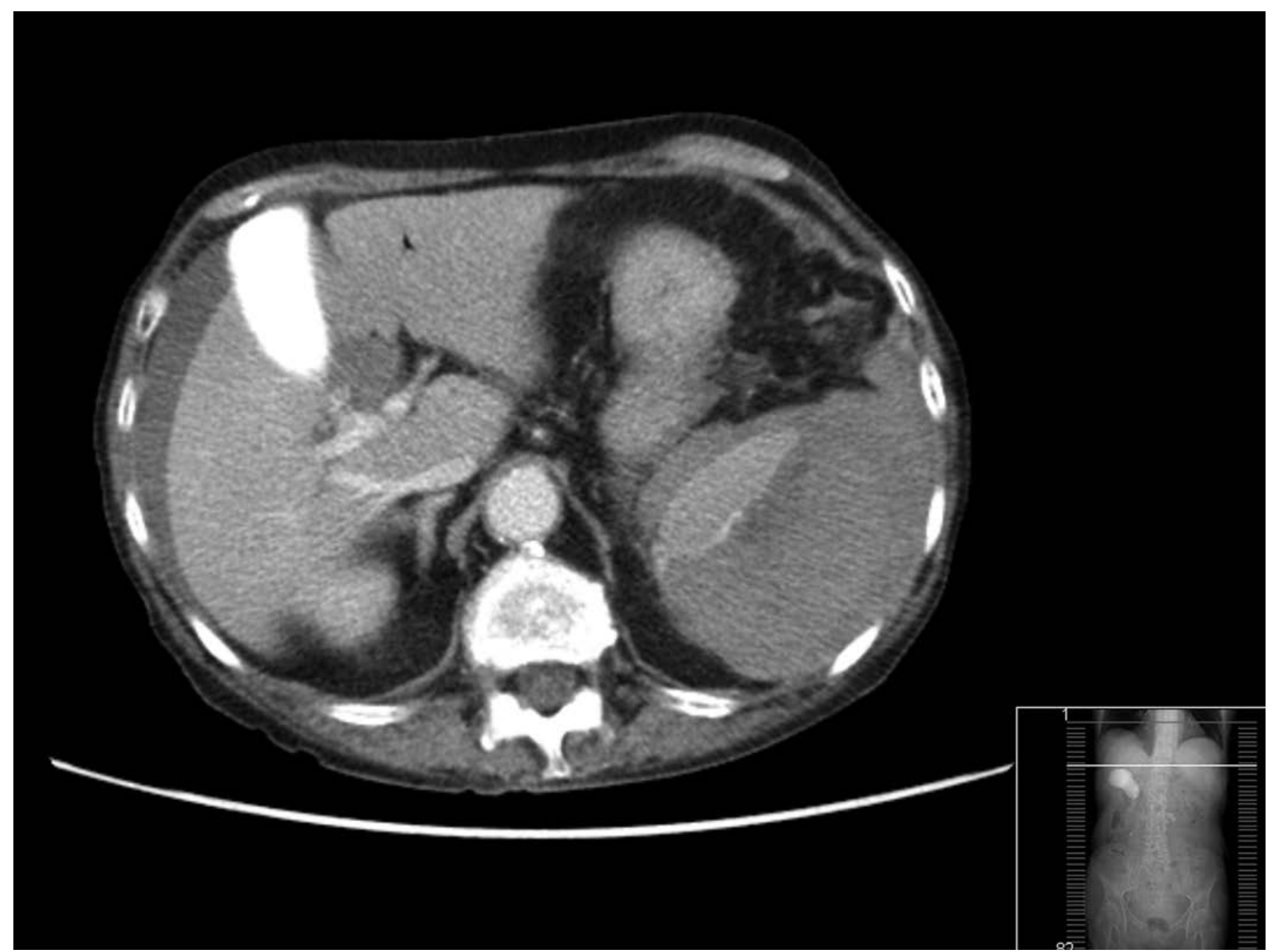

Figure 2. CT scan of patient 2 showing that there is evidence of splenic rupture with a moderate amount of free peritoneal fluid, which has a high density, mainly around the spleen (probably hemorrhagic). 
spleen was stripped, and there was a small amount of blood clot. The histology sections showed the spleen including the probable site of rupture. The white pulp showed moderate lymphoid hyperplasia. The vessels seen in the white pulp showed unremarkable features, and there was no evidence of amyloid. The red pulp of the spleen was mildly congested; however, there was no evidence of hematopoiesis. No granulomas were noted in the specimen, and there was no evidence of malignancy.

The patient had an unremarkable postoperative recovery with no complications. She received post-splenectomy vaccines and was discharged home receiving lifelong penicillin $\mathrm{V}$, with a follow-up appointment in the outpatient clinic. She had a hospital stay of 7 days. No information regarding the patient's gallstone disease is available because she was lost to follow-up.

\section{DISCUSSION}

ERCP is an important diagnostic and therapeutic tool in the management of hepatobiliary and pancreatic disorders. However, the procedure is not without risk and is associated with a number of established complications, including pancreatitis, hemorrhage, cholangitis, and bowel perforation. ${ }^{1}$ The risk of splenic injury reported in this article has important implications, especially with regard to the consent process for ERCP.

Although the exact mechanism of splenic injury after ERCP has not been established, there are a number of theories that have been proposed. One theory suggests that previous gastric surgery with the formation of adhesions between the stomach and spleen or between the spleen and parietal peritoneum may play a role, ${ }^{2,3}$ but this has not been found elsewhere despite previous laparotomy. ${ }^{4}$ The most probable explanation seems to be that such an injury is caused by traction of the short gastric vessels as a consequence of so-called bowing of the endoscope in the long position. ${ }^{5,6}$ Even the process of insufflation itself has been proposed as a potential cause of injury. ${ }^{7}$

Interestingly, in case 2 , the initial abdominal CT scan with intravenous contrast did not show any acute bleeding or blushing to suggest splenic trauma. This would suggest that the bleeding from the spleen due to endoscopic trauma was too negligible initially to show up on the scan. Negative findings from an abdominal CT scan in patients presenting with acute abdominal pain after ERCP are not sufficient to rule out suspected splenic injury. Careful clinical assessment and observation are therefore warranted in these patients before being discharged after ERCP.

Excessive manipulation of the patient required during ERCP, such as during a challenging CBD cannulation, is another potential cause of splenic injury. ${ }^{8}$ Patients with comorbidities have a higher risk of complications in general, and with regard to ERCP, there is a high theoretical risk of splenic injury in patients with liver cirrhosis and those who are taking anticoagulative medication. ${ }^{9,10} \mathrm{~A}$ lack of use of epigastric hand pressure applied to the patient by the ERCP operator (particularly when the endoscope is in the long position) could also result in a higher incidence of splenic injury. ${ }^{11}$ Most reported cases of splenic injury after ERCP have resulted in splenectomy, but there are also reports of successful conservative management of other cases involving regular clinical assessment, sequential blood tests to assess for a decrease in the hemoglobin level, and the use of procoagulant medication.

In conclusion, splenic injury is a rare but potentially fatal complication after ERCP. Clinicians must maintain a high index of suspicion for splenic trauma and keep this within the working differential diagnosis in patients presenting with acute abdominal pain after ERCP. Further evidence and discussion are required for this injury to be considered an essential part of the consenting process for ERCP.

\section{References:}

1. Vandervoort J, Soetikno RM, Tham TC, et al. Risk factors for complications after performance of ERCP. Gastrointest Endosc. 2002;56(5):652-656.

2. Kingsley D, Schermer C, Jamal M. Rare complications of endoscopic retrograde cholangiopancreatography: two case reports. JSLS. 2001;5:171-173.

3. Lo AY, Washington M, Fischer MG. Splenic trauma following endoscopic retrograde cholangiopancreatography (ERCP). Surg Endosc. 1994;8:692-693.

4. Dixon E, Graham JS, Sutherland F, Mitchell PC. Splenic injury following endoscopic retrograde cholangiopancreatography: a case report and review of the literature. JSLS. 2004;8:275277 .

5. Wu WC, Katon RM. Injury to the liver and spleen after diagnostic ERCP. Gastrointest Endosc. 1993;39:824-827. 
6. Lewis FW, Moloo N, Stiegmann GV, Goff JS. Splenic injury complicating therapeutic upper gastrointestinal endoscopy and ERCP. Gastrointest Endosc. 1991;37:632-633.

7. Furman G, Morgenstern L. Splenic injury and abscess complicating endoscopic retrograde cholangiopancreatography. Surg Endosc. 1993;7:343-344.

8. Trondsen E, Rosseland AR, Moer A, Solheim K. Rupture of the spleen following endoscopic retrograde cholangiopancreatography (ERCP). Acta Chir Scand. 1989;155:75-76.

9. Cho CL, Yuen KKY, Yuen CH, Chong LC, Chu RWS. Splenic laceration after endoscopic retrograde cholangiopancreatography. Hong Kong Med J. 2008;14:145-147.
10. Gaffney RR, Jain V, Moyer MT. Splenic injury and ERCP: a possible risk for patients with advanced chronic pancreatitis. Case Rep Gastroenterol. 2012;6(1):162-165.

11. Ong E, Bohmler U, Wurbs D. Splenic injury as a complication of endoscopy: two case reports and a literature review. Endoscopy. 1991;23:302-304.

12. Badaoui R, Ouendo M, Delcenserie R, El Kettani C, Radji M, Ossart M. Injury to the liver and spleen after diagnostic ERCP. Can J Anaesth. 2002;49(7):756-757. 